\title{
Avaliação da Modulação 4D-8PSK TCM em Canal Não Linear
}

\author{
Luciano B. C. Silva e Marcelo S. Pinho
}

\begin{abstract}
Resumo - O desempenho da modulação codificada em treliça multidimensional 4D-8PSK TCM em canal não linear é analisado por simulação, utilizando um amplificador à válvula de ondas progressivas (TWTA) comercial para o modelamento das distorções AM/AM e AM/PM. Os resultados são avaliados por meio de comparações com outras modulações, enfatizando o impacto na taxa de erro de bit (BER) e no fenômeno conhecido como Spectral Regrowth. Observa-se um aumento da margem do enlace em torno de $3 \mathrm{~dB}$ em relação às modulações antipodais, para canal linear, e uma degradação de $0,25 \mathrm{~dB}$ devido às distorções não lineares, considerando uma BER de $10^{-5}$.
\end{abstract}

Palavras-Chave-Comunicação Digital, Comunicação por Satélite, Modulação Codificada em Treliça, Canal Não Linear

Abstract - The performance of multi-dimensional Trellis Coded Modulation (4D-8PSK TCM) on nonlinear channel is investigated by computer simulation using measurements of a commercial Travelling Wave Tube Amplifier (TWTA) to model the AM/AM and AM/PM distortions. Results are evaluated by comparing with other modulation types commonly used in EESS, stressing the impact on the bit error rate (BER) and on the spectral regrowth. In comparison with antipodal modulations, it can be observed a link margin gain of $3 \mathrm{~dB}$ on linear channels and a degradation around $0,25 \mathrm{~dB}$ due to nonlinear distortion when a BER of $10^{-5}$ is considered.

Keywords - Digital Communication, Communication by Satellite, Trellis Coded Modulation, Nonlinear Channel.

\section{INTRODUÇÃO}

Sistemas de comunicação espacial, operando nas faixas regulamentadas pela União Internacional de Telecomunicações (UIT) para missões envolvendo Serviços de Exploração da Terra por Satélite (Earth Exploration Satellite Service - EESS), devem ser projetados de forma eficiente, sendo capazes de transmitir as taxas de dados cada vez mais altas provenientes de seus sensores e, simultaneamente, respeitar os requisitos regulatórios impostos por órgãos internacionais. Para esse tipo de serviço, considerando enlace de descida (Downlink) dos dados, a referência [1] atribui as faixas em: banda-S (2200$2290 \mathrm{MHz})$, banda-X (8025-8400 MHz) e banda-Ka (25.5-27 $\mathrm{GHz})$.

Atualmente, embora haja uma forte tendência para que as missões de alta taxa de dados venham a migrar para a faixa de $26 \mathrm{GHz}$, ainda prefere-se trabalhar em $8 \mathrm{GHz}$. Tal fato decorre do maior número de fornecedores de componentes e da menor complexidade técnica envolvida nessa frequência, fatores relevantes no custo do projeto.
Para orientar as agências espaciais e ao mesmo tempo padronizar novas missões, o Consultative Committee for Space Data Systems (CCSDS) apresenta recomendações de sistemas de modulação com eficiência em banda, também levando em conta outros parâmetros tais como a eficiência em potência e complexidade. Dentre essas modulações recomendadas, o documento [2] apresenta a modulação codificada em treliça multidimensional 4D-8PSK TCM, que é o foco desse trabalho.

Comparando-se com modulações antipodais de envoltória constante, comuns nesse tipo de aplicação, nota-se que o sistema de modulação 4D-8PSK TCM tem a importante característica de ser tanto eficiente em potência, devido à sua codificação convolucional inerente, quanto, em determinadas configurações, eficiente em banda, alcançando uma redução da banda espectral ocupada em virtude da técnica multidimensional. Porém, a complexidade naturalmente é o fator prejudicado, devido à necessidade tanto de um codificador a bordo, assim como de um receptor dedicado capaz de processar e decodificar os dados através de um algoritmo de Viterbi ligeiramente modificado.

Enfatizando a aplicação em questão, a principal contribuição desse trabalho é a analise do impacto das distorções causadas pela passagem do sinal modulado 4D8PSK TCM em um amplificador de alta potência (High Power Amplifier - HPA). Sabe-se que, a partir das variações na envoltória do sinal modulado, geralmente causadas pela limitação de banda, as conversões definidas como AM/AM e $\mathrm{AM} / \mathrm{PM}$, presentes em dispositivos não lineares, degradam os sinais cuja informação está contida na fase. O impacto da distorção, tanto na taxa de erro de bit como na eficiência espectral do sinal na saída do dispositivo, é analisado considerando uma TWTA comercial a ser utilizada no transmissor de dados das câmeras MUX e WFI (MWT) do satélite CBERS 3 do Instituto Nacional de Pesquisas Espaciais.

Na seção II será apresentada uma breve caracterização das distorções não lineares em sinais PSK, visto que tais sinais são parte integrante do codificador 4D-8PSK TCM. Também nessa seção é apresentado o modelo proposto assim como os coeficientes obtidos no modelamento da TWTA anteriormente mencionada. Já na seção III será descrito o funcionamento desse codificador, apoiando-se na teoria referenciada. A seção IV apresenta aspectos considerados nas simulações e os resultados. Por fim, as conclusões estão expostas na sessão V.

\section{DISTORÇÕES EM CANAL NÃO LINEAR}

A necessidade da operação das TWTAs na região não linear em satélites de sensoriamento remoto provem das altas taxas de dados empregadas nesse tipo de missão EESS. Como

Luciano B. C. Silva, Divisão de Eletrônica Aeroespacial, Instituto Nacional de Pesquisas Espaciais, São José dos Campos-SP, Brasil, E-mails: luciano.silva@inpe.br,

Marcelo S. Pinho, Divisão de Engenharia Eletrônica, Instituto Tecnológico de Aeronáutica, São José dos Campos-SP, Brasil, E-mails: mpinho@ieee.org 
consequência, existe a premissa da escolha de sistemas de modulação antipodais e com envoltória constante, reduzindo assim os efeitos das distorções não lineares.

Como o sistema 4D-8PSK TCM pode ser visto como uma modulação 8PSK com codificação e mapeamentos específicos (apresentados na próxima seção), considera-se esse tipo de sinal para a análise. Seja o sinal modulado em PSK $s(t)$ definido por

$$
s(t)=A \cos \left(2 \pi f_{p} t+\theta(t)+\varphi\right),
$$

onde $A$ é a envoltória do sinal, constante em sinais PSK, $f_{p}$ como a frequência de operação da portadora, $\theta(t)$ como a componente em fase contendo a informação e $\varphi$ como o desvio de fase constante. Porém, na prática esse sinal tem sua banda limitada para evitar interferência no canal adjacente, fazendo com que sua envoltória não seja mais constante, sendo agora definido como:

$$
x(t)=A(t) \cos \left(2 \pi f_{p} t+\theta(t)+\varphi\right) .
$$

Ao passar pelo dispositivo não linear, essa variação na envoltória gera distorções no sinal de saída, por meio de modulações não intencionais AM e PM. Dessa forma, o sinal observado na saída do dispositivo pode ser representado por

$$
y(t)=g[A(t)] \cos \left(2 \pi f_{p} t+\theta(t)+f[A(t)]+\varphi\right)(3)
$$

onde $g[A(t)]$ é a conversão $\mathrm{AM} / \mathrm{AM}$ e $f[A(t)]$ é a conversão $\mathrm{AM} / \mathrm{PM}$, que definem a não linearidade instantânea do $H P A$.

O modelamento dessas conversões de uma TWTA, dispositivo comum em satélites devido à alta geração de potência de saída, utilizado para as simulações foi o modelo de Saleh [3].

Como o ponto de operação determina o impacto das distorções no sinal, define-se o recuo (back off) de entrada (IBO) e saída (OBO) por:

$$
\begin{aligned}
& I B O=10 \cdot \log \left(P_{0, E} / P_{E}\right) \\
& O B O=10 \cdot \log \left(P_{0, S} / P_{S}\right)
\end{aligned}
$$

onde $P_{E}$ é a potência média do sinal de entrada, $P_{S}$ é a potência média do sinal de saída e $P_{O}$ é a potência máxima (na saturação) do dispositivo.

Através do IBO, atenua-se o sinal na entrada do HPA trabalhando assim em uma região mais linear do dispositivo. $\mathrm{Na}$ Seção IV será analisado o comportamento do sinal 4D8PSK TCM considerando o IBO.

O dispositivo utilizado na simulação foi modelado considerando os dados de uma TWTA TED (THALES Electron Devices) LH01 (modelo de voo), fabricada em abril/2008, que será utilizada no subsistema MWT, transmissor de dados provindos das câmeras a bordo dos satélites CBERS 3. As medidas do dispositivo, usadas como entradas para o modelo foram obtidas considerando: frequência de operação de $8200 \mathrm{MHz}$ (dentro da faixa destinada para aplicações de EESS), potência de saída na saturação: 53,2 W (típica para transmissão de canais com alta taxas de bits, ex. $100 \mathrm{Mbps)}$ e potência de entrada na saturação: $-3,3 \mathrm{dBm}$.

Outros fatores especificados do dispositivo, tais como figura de ruído, temperatura de operação, aging, retardo de grupo, etc., naturalmente, contribuiriam para uma variação nas distorções e, consequentemente, afetariam o desempenho do sistema. No entanto, as análises aqui apresentadas representam as medidas do dispositivo em uma situação padrão.

A conversão AM/AM corresponde à variação do ganho do sinal amplificado de acordo com a amplitude do sinal de entrada. Dessa forma, a condição desejada no sistema com a utilização de um par de filtros RRC (Root Raised Cosine) não é mais atingida, ou seja, ocorre um descasamento entre a forma de onda transmitida com a que o receptor espera receber, podendo também gerar uma interferência entre os símbolos (ISI), acarretando assim uma degradação do sinal que é refletida na queda da sua eficiência em potência. Além disso, outro ponto importante é a queda da eficiência espectral devido ao ressurgimento dos lóbulos laterais no espectro de potência (spectral regrowth) gerado pela conversão.

A partir da curva AM/AM medida dessa TWTA em questão, pode-se modelar a equação $g(A)$ a fim de obter seu ganho de saída variante com as flutuações na envoltória do sinal de entrada. O Modelo de Saleh, cujas equações de modelamento para uma TWTA são baseadas simplesmente em apenas dois parâmetros $\left(\alpha_{g} e \beta_{g}\right)$, para essa curva AM/AM é dado por

$$
g(A)=\frac{2,3467 A}{1+1,2619 A^{2}}
$$

onde os coeficientes ótimos $\alpha_{g}=2,3467$ e $\beta_{g}=1,2619$ são obtidos através da minimização do erro médio quadrático seguindo o procedimento de ajuste apresentado por Saleh [3]. Tal modelo mostra-se preciso e possui um valor de raiz do erro médio quadrático muito pequeno quando comparado com os pontos medidos, igual a 0,03 .

Outra distorção severa, especialmente em sistemas de modulação em fase, decorre do fato de que as variações na amplitude da portadora de entrada produzem modulações de fase não intencionais na saída do sinal amplificado. Essa forma de distorção na portadora é conhecida como conversão AM/PM. De fato, o ruído de fase aleatório provocado por esse efeito tende a ser mais degradante conforme se aumenta o nível da modulação, o que mostra a relevância dessa avaliação para sinais 4D-8PSK TCM que, a rigor, são modulados em 8PSK. Dessa forma, pode-se avaliar o real efeito da partição adotada no conjunto de sinais que sofre os impactos da não linearidade.

Assim como no modelamento anterior, a partir da curva medida de desvio de fase da TWTA em questão, também foi utilizado o modelo de Saleh [3], cujas equações de modelamento para uma TWTA também são baseadas simplesmente em apenas dois parâmetros $\left(\alpha_{f} e \beta_{f}\right)$. A equação $f(A)$ para a curva AM/PM da TWTA em questão é dada por

$$
f(A)=\frac{2,0442 A^{2}}{1+2,302 A^{2}}
$$

onde, como no caso anterior, os coeficientes ótimos $\alpha_{f}=$ 2,0442 e $\beta_{f}=2,302$ são obtidos através da minimização do erro médio quadrático aplicando o procedimento de ajuste apresentado por Saleh [3]. Assim como na curva AM/AM, observa-se uma boa precisão do modelo em questão também para essa curva de desvio de fase, ocasionando um valor de raiz do erro médio quadrático pequeno quando comparado aos pontos medidos na curva original, igual a $0,58^{\circ}$. 


\section{4D-8PSK TCM}

A técnica multidimensional do TCM, introduzida em [4] por Pietrobom et al., faz com que o 4D-8PSK TCM seja o sistema de modulação mais eficiente dentre os recomendados pelo CCSDS. Quando comparada às modulações antipodais, comuns nesse tipo de aplicação, tal sistema se revela mais eficiente tanto em banda, através de maior número médio de bits de informação transmitido durante o período do símbolo $T$ (parâmetro denominado taxa efetiva $R_{e f f}$ ), quanto em potência, através de um aumento na margem do enlace de comunicações. Entretanto, as quatro eficiências em banda apresentadas na norma [2] ( $R_{\text {eff }}$ iguais a $2,2,25,2,5$ e 2,75 bits por símbolo do canal) são inversamente proporcionais à eficiência em potência, i.e., um sistema menos eficiente transmitindo 2 bits por símbolo do canal, equivalente ao QPSK, possui maior ganho de código e, consequentemente, requer uma menor taxa $E_{b} / N_{0}$ para atingir BER especificada. Embora os sistemas considerando diferentes $R_{\text {eff }}$ sejam abordados nesse trabalho, somente foram feitas simulações com 2 bits por símbolo do canal.

A Fig. 1 apresenta os elementos do codificador para o caso específico de análise, onde $R_{\text {eff }}$ é igual a 2 bits por símbolo do canal. Basicamente, seus elementos são: conversor serial/paralelo, codificador diferencial, codificador convolucional sistemático e mapeador. As informações necessárias para a implementação de sistemas com outras taxas efetivas da modulação podem ser encontradas em [2].

$\mathrm{O}$ sistema funciona com 8 bits paralelos em sua entrada e utiliza um código convolucional sistemático, que gera o bit adicional de paridade em sua saída $z^{0}$. Logo, tal sistema possui uma taxa de modulação $R_{m}$ equivalente a $8 / 9$. Os bits de entrada acrescidos do bit de paridade entram no mapeador que entrega 12 bits na saída, equivalente as quatro constelações 8PSK que serão transmitidas sequencialmente.

Resumidamente, a fonte de dados serial provida pelo sensor passa por um conversor série/paralelo que produz em sua saída blocos de 8 bits, caracterizados pelo vetor $\mathbf{w}$, i.e., $\mathbf{w}=\left[\mathrm{w}^{1}\right.$ $\mathrm{w}^{8}$ ]. Ressalta-se a importância de uma codificação diferencial para eliminar a ambiguidade de fase em sinais PSK, efeito que pode ser gerado na recuperação do sincronismo da portadora. Por essa razão, o vetor $\mathbf{w}$ passa por um codificador diferencial que tem seu desenvolvimento apresentado em [4].

Para essa taxa de eficiência, somente as componentes $\mathrm{w}_{1}, \mathrm{w}_{5}$ e $\mathrm{W}_{8}$ são codificadas devido ao fato de estarem susceptíveis à rotação. A referência [2] apresenta as componentes susceptíveis, que devem ser codificadas, conforme a eficiência implementada.

No próximo elemento, a codificação em treliça é feita através de um codificador convolucional sistemático com taxa $R=3 / 4$ e $v=7$ (resultando em 64 estados na treliça), onde o bit de paridade $z^{0}$ é gerado em função dele mesmo e dos outros três bits de entrada $\left(z^{1}, z^{2} e z^{3}\right)$. A escolha do código a ser implementado partiu do algoritmo de busca apresentado em [4], que se baseou no critério de maximização da distância mínima euclidiana $d_{\text {free }}$ (ou ganho de código assintótico) e no menor número de vizinhos próximos $N_{\text {free }}$ O código escolhido, considerando também aspectos relacionados à rotação de fase, produz um ganho assintótico em torno de $5,5 \mathrm{~dB}$, sendo especificado pelos polinômios em octal $\mathrm{h}^{3}=050, \mathrm{~h}^{2}=024$, $\mathrm{h}^{1}=006 \mathrm{e} \mathrm{h}^{0}=103$.

Adicionalmente, na teoria de qualquer sistema TCM, sabese que através dos bits codificados o subconjunto do nível de partição em questão é selecionado, enquanto os bits não codificados selecionam os pontos dentro desses subconjuntos. Dessa forma, é interessante ressaltar que a implementação desse código convolucional de taxa $R=3 / 4$, sendo independente da eficiência $R_{\text {eff }}$ em questão, mostra o compromisso entre a eficiência em potência e banda do sistema. Ao melhorar-se a eficiência em banda introduzindo mais bits de informação $(9,10$ ou 11 bits de entrada), aumentase o número de sinais dentro do subconjunto determinado pelos bits codificados, fato que diminui a distância euclidiana entre os sinais e, consequentemente, diminui a eficiência em potência do sistema.

Já o mapeamento desses 9 bits, bloco seguinte no diagrama da Fig.1 segue a forma ótima com a qual o sinal foi particionado. Esse mapeamento varia de acordo com $R_{\text {eff }} \mathrm{e}$ sendo que mais informações, aqui omitidas, podem ser encontradas em [2]. A saída apresenta os 12 bits que serão transmitidos sequencialmente em símbolos 8PSK pelo modulador.

De forma sucinta, o processo de decodificação suave é feito com o auxilio de uma treliça auxiliar, que faz a função da decodificação do subconjunto $\left(1^{\circ}\right.$ passo $)$ e alimenta o algoritmo de Viterbi tradicional ( $2^{\circ}$ passo) para a busca do caminho com menor métrica entre as sequências permitidas pela treliça principal. A complexidade da decodificação cresce de acordo com o número de bits não codificados envolvidos (maior eficiência em banda), aumentando o número de ramos paralelos na treliça.

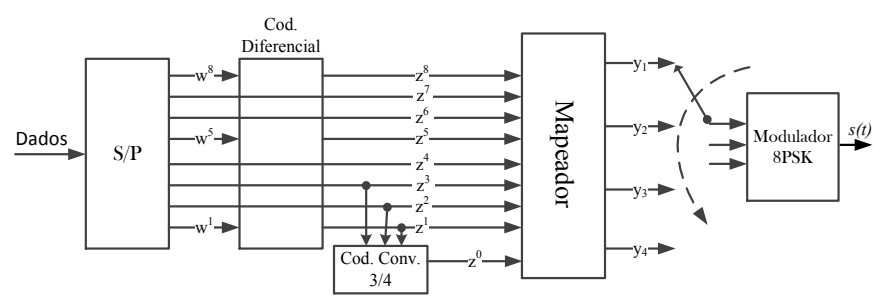

Fig. 1. Codificador 4D-8PSK TCM para $R_{\text {eff }}$ de 2 bits por símbolo do canal [2]..

\section{Simulação E Resultados}

\section{A. Sistema de Simulação}

A Fig. 2 mostra diagrama de blocos contendo todos os elementos utilizados na simulação do sistema de comunicação equivalente passa baixas, onde as setas indicam sinal complexo. As simulações foram feitas utilizando os softwares SystemVue 2011.10 da Agilent Technologies e MATLAB R2009a da MathWorks.

O diagrama inicia com uma fonte de dados aleatória sendo que, para a simulação, de forma a prover uma estatística suficiente para as análises de BER, o número de bits de informação de $10^{8}$ foi considerado. Passando pelo codificador descrito na seção anterior, a partir daqui, considera-se um sistema de transmissão 8PSK típico, visto que $y_{1}, y_{2}, y_{3}$ e $y_{4}$ são transmitidos sequencialmente. Dessa forma, o sinal complexo em banda base na saída do modulador 8PSK natural é dado por

$$
s_{i, n}=A \exp \left(j \theta_{n}\right)
$$


Em seguida as componentes fase e quadratura desse sinal complexo são convoluídas pelo filtro RRC com fator de roll off $\alpha=0,5$. Devido à causalidade do filtro, um atraso de $8 . T$ foi implementado, onde $T$ é o período do símbolo. Nesse ponto, como mencionado anteriormente, tem-se a envoltória do sinal variando devido à resposta impulsiva do filtro, logo, na saída do amplificador de alta potência $H P A$ o seguinte sinal é observado

$$
y_{l}(t)=g[A(t)] \cos (j \theta(t)+j f[A(t)]+j \varphi)
$$

onde as funções $g($.) e $f($.) caracterizam as distorções AM/AM e AM/PM respectivamente, tendo seu modelamento descrito na seção II, assim como as outras componentes da equação. Admitindo-se o canal AWGN, o sinal na entrada receptor coerente é caraterizado como

$$
r_{l}(t)=c y_{l}(t)+n(t)
$$

sendo que a constante complexa $c$ ajusta a fase do sinal recebido de modo a compensar o desvio médio de fase do HPA em questão, sendo dependente do IBO aplicado. Na sequência o sinal recebido $r_{l}(t)$ convolui com o filtro casado RRC com fator de roll off $\alpha=0,5 \mathrm{e}$, por fim, antes de entrar no decodificador de Viterbi e assumindo uma sincronização de símbolos perfeita, o sinal é amostrado em $t=n T$ fornecendo o símbolo 8PSK a ser estimado $z_{n}=z(n T)$.

$\mathrm{Na}$ decodificação, apoiado no que já foi apresentado na sessão anterior, os passos do decodificador de Viterbi de 64 estados deslocam-se a cada quatro símbolos de entrada $z_{n}$. Na implementação, foram usados 42 passos para decisão dos 4 primeiros símbolos inseridos no decodificador. Sequencialmente essa janela de 42 passos desliza e decodifica os próximos 4 símbolos $z_{n}$, repetindo esse processo sucessivamente até que sejam decodificados todos os bits gerados pela fonte do sistema. As métricas assim como os pontos de sinal escolhidos, que possuem menor distância euclidiana, para o decodificador de Viterbi são fornecidos pela treliça auxiliar montada.

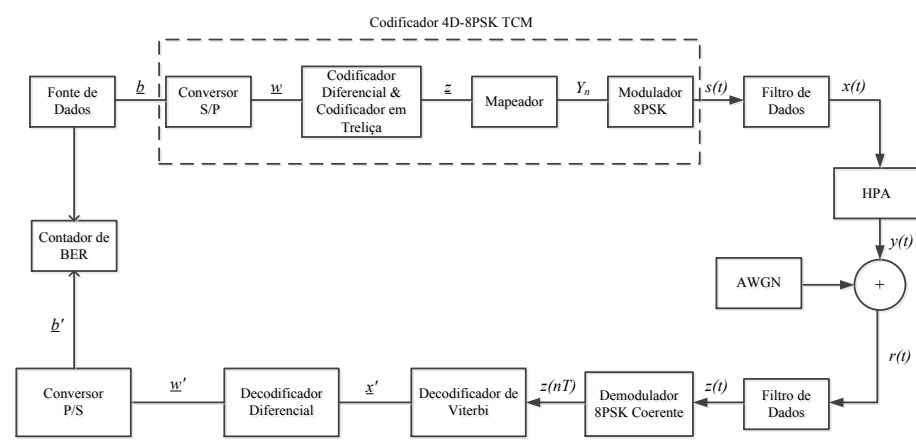

Fig. 2. Sistema de comunicação simulado.

\section{B. Desempenho Sinais PSK em Canal Não Linear}

Antes de avaliar os efeitos do sinal 4D-8PSK TCM percorrendo um dispositivo não linear, é necessário verificar o que ocorre em modulações de especial interesse ao trabalho, estabelecendo assim mais um critério comparativo entre as possíveis escolhas de sistemas de modulação.

Com base nessas informações, o objetivo dessa seção será analisar os efeitos da não linearidade na modulação QPSK, que serve de referência para o sistema TCM, e 8PSK que é o modulador utilizado no sistema de modulação 4D-8PSK
TCM, sendo outra contribuição desse trabalho. Logo, em resumo, nessa seção será considerado o sistema de comunicação da Fig. 2 sem essa prévia codificação e mapeamento, sendo demodulado coerentemente e admitindo uma perfeita compensação do desvio fase gerada pela TWTA. Conforme recomendação do CCSDS, para eliminar as distorções causadas pela filtragem, o par de filtros RRC com $\alpha$ $=0,5$ foi utilizado na limitação de banda.

Na Fig. 3 a curva de BER de um sinal QPSK e de um sinal 8PSK passando pela TWTA TED LH01 modelada, com seu ponto de operação saturado $(I B O=0)$, é exposta. Observa-se que, na condição de perfeita recuperação de fase e símbolo, as distorções decorrentes da não linearidade não degradam significativamente o sinal QPSK, i.e. em torno de $0,5 \mathrm{~dB}$ considerando uma BER de $10^{-5}$. Conjectura-se que essa degradação seja causada principalmente pela perda da condição de casamento entre os filtros RRC de transmissão e recepção devido à conversão $\mathrm{AM} / \mathrm{AM}$. Quanto às distorções de fase, causadas pela conversão anteriormente definida $\mathrm{AM} / \mathrm{PM}$, pode-se considerar que não são tão severas quanto em modulações de ordens mais altas, como por exemplo, o 8PSK, também apresentado.

Outra observação importante a ser feita é o aumento significativo da degradação em sistemas 8PSK em detrimento à simulação com modulação QPSK. Isso ocorre devido à maior susceptibilidade do 8PSK ao ruído aleatório de fase na constelação, gerado pela conversão $\mathrm{AM} / \mathrm{PM}$, com degradações que podem superar a $2 \mathrm{~dB}$, adotando uma BER de $10^{-5}$.

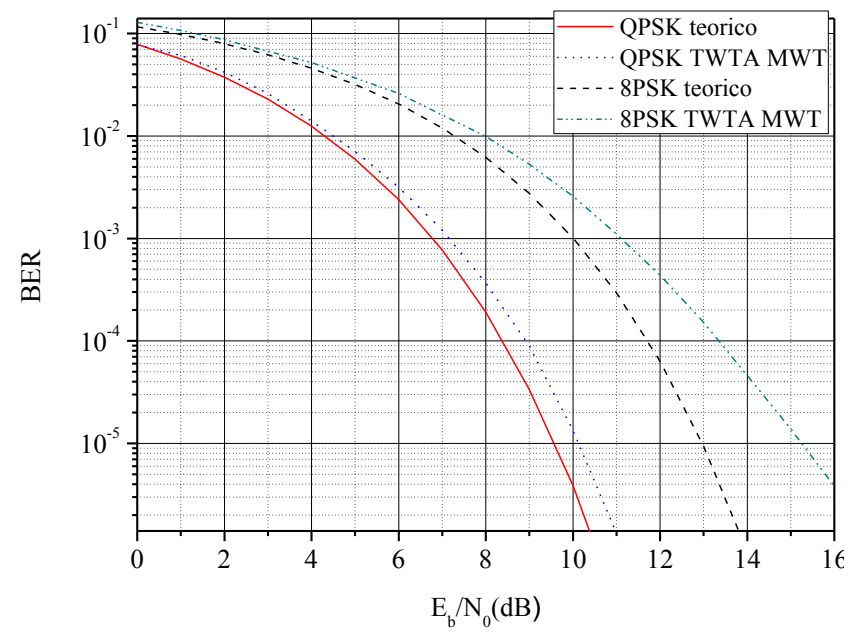

Fig. 3. Curva de BER sistemas PSK com TWTA TED LH01 modelada

Os resultados aqui apresentados servem como parâmetro para uma avaliação do impacto das distorções devido à não linearidade em sinais de modulação codificada. Pela teoria apresentada, foi visto que as partições tem o objetivo de aumentar a distância euclidiana entre os pontos de sinal o que, necessariamente, teriam impacto na susceptibilidade ao ruído de fase causado pela distorção AM/PM.

\section{Desempenho Sinais 4D-8PSK TCM em Canal Não Linear}

Porém, ainda é necessário saber o desempenho do sinal 4D-8PSK TCM com $R_{\text {eff }}=2$ bits por símbolo do canal percorrendo um canal não linear. Partindo da premissa de que a escolha desse sistema surge da necessidade de um maior ganho na margem do enlace de um sistema de comunicação utilizando modulação antipodal, provavelmente com a TWTA saturada, é necessário saber se as distorções devido ao amplificador irão aumentar com o emprego da codificação. Ou 
seja, para que o sistema seja também viável nesse tipo de canal, seu calculo de enlace não deve ter sua perda tecnológica, ou perda de implementação, que prevê e quantifica essas distorções, significativamente aumentada.

Começando pela análise quanto ao espectro transmitido, foram feitas simulações variando o IBO na entrada da TWTA TED LH01 (modelo de voo) modelada, conforme definido na seção II. A Fig 4 apresenta a densidade espectral de potência DEP normalizada para o caso de IBO igual a 0 (saturação).

Foi observado o aumento significativo das distorções devido à não linearidade no espectro do sinal transmitido. Entretanto, o resultado importante trazido pela Fig. 4 é que no pior caso (saturação) a máscara do SFCG (adotada como critério de eficiência em banda em [2]) é cumprida. Isso remete ao fato de que, apesar do 8PSK não ser tão eficiente em canais não lineares como em modulação cujas transições de $180^{\circ}$ não são permitidas entre estados consecutivos, como por exemplo, os recomendados pelo CCSDS OQPSK e MSK, o filtro RRC com $\alpha=0,5$ no transmissor provê, se tratando de missões com vários canais, uma limitação de banda suficiente para reduzir a interferência no canal adjacente.

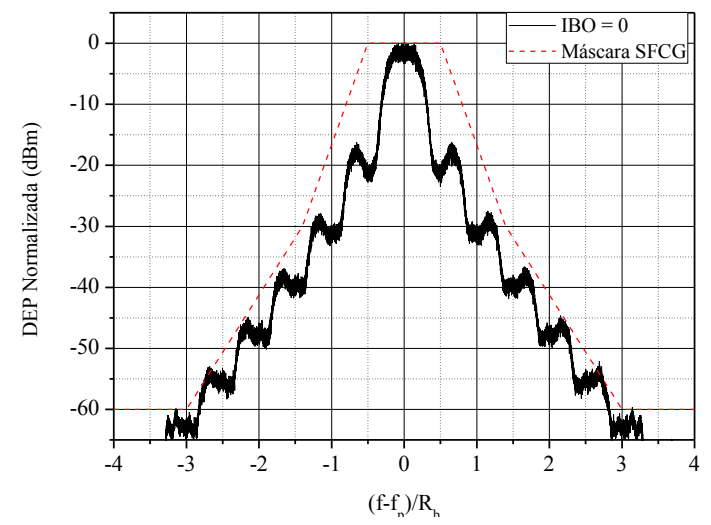

Fig. 4. Densidade Espectral de Potência do sinal 4D-8PSK TCM IBO=0.

Considerando a eficiência em potência, o segundo importante resultado observado através da Fig. 5 é a presença de uma pequena degradação, em torno de $0,25 \mathrm{~dB}$ para uma BER de $10^{-5}$, menor do que a obtida para os sistemas modulados da seção anterior. Isso decorre do fato de como foram feitas as partições, detalhadas em [4], tornando o sistema menos susceptível ao ruído de fase em relação ao QPSK (degradação em torno de $0,5 \mathrm{~dB}$ para uma BER de $10^{-5}$ ) e principalmente ao 8PSK (degradação maior que $2 \mathrm{~dB}$ para uma BER de $10^{-5}$ ). Essa conclusão reforça o emprego do TCM como uma boa escolha para esse tipo de aplicação.

Em situações práticas, esse aumento de margem pode ser traduzido no uso de TWTAs menos potentes, ou até mesmo no uso SSPAs, que geralmente possuem menor ganho além de características diferentes. Outra vantagem é a possibilidade de redução da figura mérito de $G / T$ do sistema de recepção, implicando em estações terrenas de menor custo, fator de relevante em missões EESS de transmissão de imagens.

Finalizando a seção, é importante mencionar que os efeitos dessas distorções variam de acordo com o sistema de recepção implementado, sendo esses resultados considerados como um limitante inferior devido à perfeita recuperação de fase e símbolo. Mais ainda, tais distorções variam de acordo com a filtragem empregada e com o dispositivo em questão.

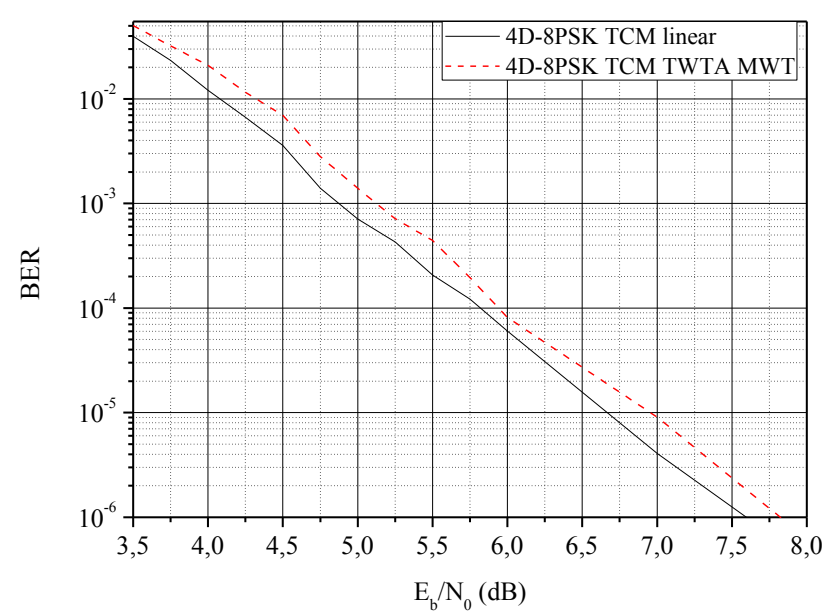

Fig. 5. Curva de BER 4D-8PSK TCM IBO=0.

\section{CONCLUSÕES}

Este trabalho apresentou o comportamento do sistema de modulação recomendado pelo CCSDS para missões de EESS 4D-8PSK TCM, com $R_{\text {eff }}=2$ bits por símbolo, em um canal não linear. Para isso, uma simulação envolvendo os conceitos aplicados na codificação desse sinal, nos diversos elementos de um sistema de comunicação espacial e, por fim, na decodificação, que é realizada a partir de um algoritmo Viterbi apoiado em uma treliça auxiliar, foi implementada. Já o modelamento do canal não linear, realizado utilizando o modelo de Saleh [3], foi concebido a partir das curvas AM/AM e desvio de fase medidas de uma TWTA comercial TED LH01 (modelo de voo), a ser utilizada no transmissor de dados do satélite CBERS 3.

Os resultados obtidos demonstraram que o sistema de modulação 4D-8PSK TCM, limitado em banda por filtro RRC de $\alpha=0,5$, é eficiente tanto em banda quanto em potência, conforme os critérios que foram estabelecidos no decorrer do texto. Mais ainda, que esse sistema de modulação é menos susceptível às distorções não lineares em relação a sistemas de modulação comuns nessa aplicação, tais como QPSK ou 8PSK, fato atribuído devido à forma como o conjunto de sinais foi particionado. Sob tal conclusão, atesta-se que o aumento da margem no enlace de comunicações decorrente do uso desse sinal torna-se ainda maior, quando comparada ao sinal QPSK, em ambiente não linear.

\section{AGRADECIMENTOS}

À Agilent Technologies do Brasil pelas licenças educacionais do pacote de software EESOF, do qual faz parte o SystemVue 2011 utilizado como ferramenta para esse trabalho, gentilmente cedidas.

\section{REFERÊNCIAS}

[1] International Telecommunication Union (ITU), Handbook EESS., 2011.

[2] Consultative Committee dor Sapace Data System (CCSDS), CCSDS 413.0-G-2-Bandwidth Efficient Modulations Green Book, Outubro 2009.

[3] A. A. M. Saleh, "Frequency-Independent and Frequency-Dependent Nonlinear Models of TWT Amplifiers", IEEE Transactions on Communications, v. COM-22, N 11, pp. 1715-1720, 1981.

[4] S. Pietrobon; A. Lafanachère; G. Ungerboeck; D. J. Costello, "TrellisCoded Multidimensional Phase Modulation", IEEE Transactions of Information Theory, v. 36, N 1, pp. 63-89, 1990.

[5] D. Servant, "Design and Analysis of Multidimensional Trellis Coded Demodulator". Master's Thesis, KTH Department of Signals, Sensors, Systems, Stockholm, Sweden, June 2006. 